\title{
Trefoil Factor 1 Suppresses Stemness And Enhances Chemosensitivity Of Pancreatic Cancer
}

Junpei Yanaguchi ( $\square$ jumpei@med.nagoya-u.ac.jp )

(1) Division of Surgical Oncology, Department of Surgery, Nagoya University Graduate School of Medicine

\section{Toshio Kokuryo}

(1) Division of Surgical Oncology, Department of Surgery, Nagoya University Graduate School of Medicine

\section{Yukihiro Yokoyama}

(1) Division of Surgical Oncology, Department of Surgery, Nagoya University Graduate School of Medicine

\section{Shunsuke Oishi}

(2) Institute of Transformative Bio-Molecules, Nagoya University

\section{Masaki Sunagawa}

(1) Division of Surgical Oncology, Department of Surgery, Nagoya University Graduate School of Medicine

\section{Takashi Mizuno}

(1) Division of Surgical Oncology, Department of Surgery, Nagoya University Graduate School of Medicine

\section{Shunsuke Onoe}

(1) Division of Surgical Oncology, Department of Surgery, Nagoya University Graduate School of Medicine

\section{Nobuyuki Watanabe}

(1) Division of Surgical Oncology, Department of Surgery, Nagoya University Graduate School of Medicine

\section{Atsushi Ogura}

(1) Division of Surgical Oncology, Department of Surgery, Nagoya University Graduate School of Medicine

\section{Tomoki Ebata}

(1) Division of Surgical Oncology, Department of Surgery, Nagoya University Graduate School of Medicine

\section{Research Article}


Keywords: pancreatic cancer, chemosensitivity, cancer stem cell, trefoil factor, epithelialmesenchymal transition

Posted Date: February 23rd, 2022

DOI: https://doi.org/10.21203/rs.3.rs-1294391/v1

License: (1) This work is licensed under a Creative Commons Attribution 4.0 International License. Read Full License 


\section{Research Article}

\section{Trefoil factor 1 suppresses stemness and enhances chemosensitivity of pancreatic cancer}

Authors: Junpei Yamaguchi ${ }^{(1) *}$, Toshio Kokuryo ${ }^{(1)}$, Yukihiro Yokoyama ${ }^{(1)}$, Shunsuke Oishi ${ }^{(2)}$, Masaki Sunagawa $^{(1)}$, Takashi Mizuno ${ }^{(1)}$, Shunsuke Onoe ${ }^{(1)}$, Nobuyuki Watanabe ${ }^{(1)}$, Atsushi Ogura $^{(1)}$, and Tomoki Ebata ${ }^{(1)}$

(1) Division of Surgical Oncology, Department of Surgery, Nagoya University Graduate School of Medicine, Nagoya, Japan

(2) Institute of Transformative Bio-Molecules, Nagoya University, Nagoya, Japan

Key words: pancreatic cancer, chemosensitivity, cancer stem cell, trefoil factor, epithelialmesenchymal transition

\section{Corresponding Author:}

Junpei Yamaguchi, MD, PhD

Email: jumpei@med.nagoya-u.ac.jp

Surgical Oncology, Nagoya University Graduate School of Medicine

65 Tsurumai-cho, Showa-ku, Nagoya

Aichi, Japan 466-8550

Phone: $+81-52-744-2222$

Fax: $+81-52-744-2230$

Disclosure: The authors have declared that no conflict of interest exists. 


\section{Abstract}

Pancreatic cancer is one of the most lethal malignancies, partly due to its high resistance to conventional chemotherapy. The aim of this study is to investigate the association between chemoresistance and trefoil factor family 1 (TFF1), a tumorsuppressive protein in pancreatic carcinogenesis. To investigate the role of TFF1 in human and mice, specimens of human pancreatic cancer and genetically engineered mouse model of pancreatic cancer (KPC/TFF1KO; Pdx1-Cre/ LSL-KRAS G12D/ LSL$\mathrm{p} 53^{\mathrm{R} 172 \mathrm{H} /} \mathrm{TFF}^{-/-}$) were analysed. The expression of TFF1 in cancer cells was associated with better survival of the patients who underwent chemotherapy, and the deficiency of TFF1 increased EMT of cancer cells and deteriorated the benefit of gemcitabine in mice. To explore the efficacy of TFF 1 treatment, recombinant and chemically synthesized TFF1 were administered to pancreatic cancer cell lines and mouse models. TFF1 inhibited gemcitabine-induced EMT, Wnt pathway activation and cancer stemness, eventually increased apoptosis of pancreatic cancer cells by gemcitabine. Combined treatment of gemcitabine and TFF1 arrested tumor growth and resulted in the better survival of mice. These results indicate that TFF1 can contribute to establishing a novel strategy to treat pancreatic cancer patients by enhancing chemosensitivity. 


\section{Introduction}

Although treatments for pancreatic cancer, including surgical resection,

irradiation and chemotherapy, have been developed, the efficacy of these treatments and the survival of patients remain undesirable. Tumor chemoresistance hampers the development of treatments. For instance, although multidrug chemotherapy, such as FOLFIRINOX and gemcitabine/nab-paclitaxel, has contributed to the improvement of patient survival, the incidence of adverse effects is high, and the response rate remains as low as $30 \%{ }^{1,2}$.

The mechanisms of chemoresistance have been investigated, and epithelialmesenchymal transition (EMT) and cancer stemness were shown to contribute to therapeutic resistance ${ }^{3-6}$. EMT is characterized by the conversion of cancer cells from an epithelial phenotype to a mesenchymal phenotype and thus plays a role in various processes during the progression of malignant diseases such as invasion, dissemination to distant organs, and resistance to antitumor drugs. EMT can be triggered by various microenvironmental stimuli, including not only hypoxia and low $\mathrm{pH}$ but also autocrine and paracrine factors. Such factors induce cancer cells to develop a mesenchymal phenotype by regulating zinc-finger proteins (such as Snail, Slug, and ZEB1) and various signalling pathways, including the Wnt cascade and PI3A/AKT axis ${ }^{7}$. Of note, 
EMT of cancer cells can also be induced by anticancer agents; thus, long-term chemotherapy results in acquired drug resistance of the tumor ${ }^{8}$. In addition, EMT has been associated with stemness ${ }^{9,10}$, and cancer stem cells (CSCs) also show antitherapeutic effects. Given this evidence, treatments that inhibit EMT and the stemness of cancer cells could be an attractive approach. However, EMT- and stemnessinhibiting therapy remains challenging and has not been established.

TFF1 is a secreted protein that exhibits a tumor-suppressive role in pancreatic carcinogenesis by inhibiting malignant transformation of premalignant lesions, such as pancreatic intraepithelial neoplasms (PanIN) ${ }^{11}$. Interestingly, TFF1 is expressed in pancreatic duct glands (PDGs), the progenitor-cell niche of the pancreatic epithelium ${ }^{12}$, suggesting a possible association between TFF1 and stem cells. Here, we show that TFF1 can inhibit EMT and stemness, thus enhancing the chemosensitivity of pancreatic cancer. These results could provide a fundamental basis for novel cancer treatments that could have a significant effect on disease outcome. 


\section{Materials and Methods}

\section{Human samples}

Human pancreatic samples were obtained from patients who underwent pancreatectomy for pancreatic cancer $(n=91)$ at Nagoya University Hospital in accordance with the guidelines of the institutional review board at Nagoya University. The informed consent was obtained from all patients. Among these patients, the survival of those who suffered postoperative recurrence of the disease and received chemotherapy $(\mathrm{n}=46)$ was analyzed.

\section{Histology and immunohistochemistry}

The methods for IHC are described in our previous report ${ }^{11}$. In short, specimens were fixed overnight in 10\% formalin/phosphate-buffered saline. Histological analysis was performed on $4-\mu \mathrm{m}$ paraffin-embedded sections. IHC was performed using the antibodies shown in Supplemental Table 1. The sections were incubated overnight at $4^{\circ} \mathrm{C}$ with each primary antibody and incubated for $1 \mathrm{~h}$ at room temperature with secondary antibodies. Proteins were visualized using EnVision Detection Systems (Dako Japan, Tokyo, Japan). The slides were counterstained with hematoxylin. Ten randomly selected nonoverlapping fields of view (x200 microscopic 
fields) in the stained sections were imaged using a Keyence BZ-X800 (Keyence, Japan) for assessment. Analyses were performed with publicly available ImageJ software from the National Institutes of Health (NIH).

\section{Cell culture}

The methods for cell culture are described in our previous report ${ }^{11}$. In short, human pancreatic cancer cell lines (Panc1, KLM1, KP4, and PK9) were obtained from the Cell Resource Center for Biomedical Research, Institute of Development, Aging and Cancer, Tohoku University, Japan. All cell lines were cultured in RPMI 1640 medium (Invitrogen Life Technologies, Carlsbad, CA) with 10\% heat-inactivated fetal bovine serum (Equitech-Bio, Inc., Kereville, TX) at $37^{\circ} \mathrm{C}$ in a humidified atmosphere with $5 \%$ $\mathrm{CO}_{2}$.

\section{Transfection of TFF1 overexpression plasmid}

A TFF1 overexpression plasmid or a control plasmid was transfected into pancreatic cancer cell lines using a CUY21EDIT ver 2.10 electroporation system (BEX, Tokyo, Japan) according to the manufacturer's protocol. After transfection, cancer cell lines were incubated for 48 hours and then analyzed. Under gemcitabine treatment, cells 
were transfected, incubated for 24 hours, treated with gemcitabine for 48 hours and then analyzed.

The synthesis of human TFF1

Synthetic human TFF1 (shTFF1) was prepared by automated Fmoc solid phase peptide synthesis using CS136XT (CSbio, Menlo Park, CA) and Wang polystyrene resin (Merck, DE). A brief summary of the utilized synthesis protocols: Fmoc deprotections were performed with $20 \%$ piperidine in $N, N$-dimethylformamide (DMF, $2 \times 8$ min). Coupling was performed with Fmoc-amino acid (4.0 equiv relative to resin substitution), $O-\left(1 \mathrm{H}-6\right.$-chlorobenzotrazol-1-yl)- $N, N, N^{\prime}, N^{\prime}$-tetramethyluronium hexafluorophosphate (3.9 equiv) and $N$-methylmorpholine (8.0 equiv) in DMF for 60 min. If required, the coupling step was repeated once (double coupling). The obtained dry resin was placed in a glass vial, a mixture of 97:1:2 trifluoroacetic acid (TFA):triisopropylsilane: $\mathrm{H}_{2} \mathrm{O}(15 \mathrm{~mL} / \mathrm{g}$ resin) was added, and the suspension was shaken for $2 \mathrm{~h}$. The resin was removed by filtration, and the filtrate was placed in a plastic centrifugal tube $(40 \mathrm{~mL})$. Then, volatiles removed under reduced pressure. The residue was washed with diethyl ether (ca. $15 \mathrm{~mL} / \mathrm{g}$ resin). The crude material was purified by reversed-phase high-performance liquid chromatography (HPLC) on a 
Phenomenex Jupiter C18 column (5 $\mu \mathrm{m}, 300 \AA$ A pore size, $30 \mathrm{~mm}$ I.D. x $250 \mathrm{~mm}$ ) with Milli-Q- $\mathrm{H}_{2} \mathrm{O}$ containing $0.1 \%$ TFA and HPLC grade $\mathrm{CH}_{3} \mathrm{CN}$ containing $0.1 \%$ TFA as the mobile phase. The purified reduced peptide was denatured using denatured buffer containing $1 \mathrm{M} \mathrm{Gdn} . \mathrm{HCl}+0.1 \mathrm{M}$ HEPES buffer, $\mathrm{pH} 7.0$ (1.5 mM peptide concentration). The solution was diluted with a 9-fold volume of folding buffer containing $0.1 \mathrm{mM}$ phosphate buffer ( $\mathrm{pH} 8.0$ ) with $10 \%$ dimethyl sulfoxide and stirred for 24 hours at ambient temperature. Refolded shTFF1 was obtained after HPLC purification on a Phenomenex Jupiter C18 column ( $5 \mu \mathrm{m}, 300$ Å pore size, 10 mm I.D. x $250 \mathrm{~mm}$ ) and lyophilization.

Cell treatment by TFF1 protein

Recombinant human TFF1 (rhTFF1) and mouse TFF1 (rmTFF1) were purchased from Peprotech (Cranbury, NJ). Synthesized human TFF1 (shTFF1) was provided by Craftide (Nagoya, Japan). Human pancreatic cancer cell lines were plated in 6-well plates and then treated with gemcitabine and/or TFF1 (either rhTFF1 or shTFF1) for 36 hours and then analyzed. 
Cell proliferation assay

The relative cell number was assessed using a WST-1 cell proliferation assay system (Cell Proliferation Reagent WST-1; Roche, Indianapolis, IN). The cells were transfected with plasmid and seeded in a 96 -well plate $\left(5 \times 10^{3}\right.$ cells per well) or seeded and then treated with gemcitabine and/or shTFF1. After 72 hours of incubation, the medium was replaced with fresh medium containing $10 \mu \mathrm{l}$ of WST-1 reagent. Relative cell numbers were calculated by measuring the absorbance of each well at $450 \mathrm{~nm}$.

\section{Western blotting}

Proteins were extracted from cultured cells using Laemmli sample buffer

(Wako, Japan). The proteins were subjected to $10 \%$ or $15 \%$ SDS-polyacrylamide electrophoresis and transferred onto polyvinylidene difluoride (PVDF) membranes (Immobilon; Millipore, Billerica, MA). The membranes were incubated with the primary antibodies listed in Supplemental Table 2. Horseradish peroxidase (HRP)conjugated anti-rabbit IgG (1:1,000, Cell Signaling Technology) and HRP-conjugated anti-mouse IgG (1:1,000, Cell Signaling Technology) were used as the secondary antibodies. Protein expression was detected using Pierce Western Blot Substrate (Thermo, Rockford, AZ). 


\section{Real-Time PCR}

RNA was extracted from cell lines using a QIAcube (Qiagen, Hilden,

Germany) according to the manufacturer's protocols. cDNA was synthesized using a High Capacity cDNA Reverse Transcription kit (Applied Biosystems, South San Francisco, CA). Real-time PCR assays were performed using a 7300 Fast Real-Time PCR System (Applied Biosystems). Each reaction was performed in a $10 \mu \mathrm{l}$ mixture containing TaqMan universal PCR master mix (Applied Biosystems). The TaqMan probes and primers were obtained from Applied Biosystems (listed in Supplemental Table 3). In each evaluation, the relative expression of the gene of interest was normalized to that of the $18 \mathrm{~S}$ internal control.

Apoptosis assay

Apoptosis studies were performed using the Muse Annexin V and Dead Cell Kit (Millipore, Darmstadt, Germany) according to the manufacturer's protocol. The cells were seeded in 6-well plates, and the apoptotic rates were then analyzed after the treatment. 
Flow cytometry

Cells were harvested and rinsed twice with PBS and incubated with antibodies against CD133 (BD Pharmingen, clone: W6B3C1) and Hoechst 33342 (Invitrogen, Waltham, MA) for one hour at room temperature. After the washing, the cells were resuspended in PBS. Hoechst-positive cells were gated and analyzed by flow cytometry (FACSCanto 2, BD).

Animals

Pdx1-Cre (stock number: 014647), LSL-p53 ${ }^{\mathrm{R} 172 \mathrm{H}}$ (stock number: 008652), and LSL-KRAS $^{\mathrm{G} 12 \mathrm{D}}$ (stock number: 008179) mice were purchased from the Jackson Laboratory (Bar Harbor, ME, USA). TFF1-KO (KO first allele, allele name: $\mathrm{Tff}^{\mathrm{tm}}{ }^{\text {1a(EUCOMM)Wtsi }}$ ) mice were purchased from the IMPC (International Mouse Phenotyping Consortium). Pdx1-Cre, LSL-p53 ${ }^{\mathrm{R} 172 \mathrm{H}}$, and LSL-KRAS ${ }^{\mathrm{G} 12 \mathrm{D}}$ mice were bred with TFF1-KO mice to generate mice with the following genotypes: Pdx1-Cre; LSL-KRAS $^{\mathrm{G} 12 \mathrm{D}}$; LSL-p53 ${ }^{\mathrm{R} 172 \mathrm{H}}$; and TFF1-KO (KPC/TFF1KO). Murine recombinant TFF1 (mrTFF1) was purchased from Peprotech (Cranbury, NJ). Gemcitabine (100 $\mathrm{mg} / \mathrm{kg}$, intraperitoneal injection, twice per week) and mrTFF1 (1 $\mu \mathrm{g} / \mathrm{mouse}$, subcutaneous injection, twice per week) were administered at the age of two months. 
The mice were sacrificed when they became moribund, and their pancreatic samples were harvested for analysis.

Xenograft mouse model

Male BALB/c nude mice ( 8 weeks old and weighing 20-25 g) were purchased from SLC Japan (Nagoya, Japan). Panc1 cells $\left(1 \times 10^{7}\right)$ were inoculated into the femoral area of each mouse. After 10 days, the mice were randomly divided into three groups (five mice per group) and were treated with PBS, gemcitabine (40 mg/kg) or sTFF1 ( $1 \mu \mathrm{g} /$ mouse) twice per week for 4 weeks. Tumor growth was assessed by measuring the volume (in $\mathrm{mm}^{3}$ ). The volume was calculated using the following equation: $\left(\mathrm{L} \times \mathrm{W}^{2}\right) / 2$, where $\mathrm{L}$ is the tumor length and $\mathrm{W}$ is the tumor width.

\section{Statistics}

All data are presented as the mean $\pm \mathrm{SD}$ or as box-and-whisker plots (centerline, median; box limits, upper and lower quartiles; whiskers, 1.5x interquartile range; points, outliers) as appropriate. Differences were tested for significance by ANOVA. The survival of humans and mice was calculated using the Kaplan-Meier method, and differences in the survival curves were compared using the log-rank test. 
All statistical analyses were performed using the Statistical Package for the Social Sciences (SPSS) ver. 24 (SPSS, Inc., Chicago, IL) or publicly available software R. A P value $<0.05$ was considered statistically significant.

Study approval

Human samples were collected and analyzed in accordance with approval from the Institutional Review Board of Nagoya University (approved number; 2017-0080). All animal experiments were conducted in accordance with approval from the Institute for Laboratory Animal Research, Nagoya University Graduate School of Medicine (approved number; 20328). 


\section{Results}

TFF1 inhibits EMT of pancreatic cancer in vivo.

Although we found that TFF1 functions as a tumor suppressor in pancreatic premalignant lesions, it is unclear whether it has the same function in mature malignant tumors in vivo. To clarify this, we employed a mouse model of pancreatic cancer development, KPC mice (Pdx1-Cre; LSL-KRAS ${ }^{\mathrm{G} 12 \mathrm{D}}$; LSL-p53 ${ }^{\mathrm{R} 172 \mathrm{H}}$ ), which were bred with TFF1KO mice to generate KPC/TFF1KO mice, and the tumor characteristics including EMT were compared between these mouse models. The proliferative activity (Ki67) of tumor cells was lower in the KPC/TFF1KO mice (Figure 1A, B), whereas the labelling index of the EMT markers Snail (Figure 1C, D) and ZEB1 (Figure 1E, F) was significantly higher in the KPC/TFF1KO mice than the other mice. These results are consistent with the concept that the attenuation of proliferation is one of the hallmarks of $\mathrm{EMT}^{13,14}$. Since EMT is thought to promote invasion and metastasis of pancreatic cancer, the incidence of distant metastasis was then analyzed. Although liver and lung metastases were found and histologically confirmed in some mice (Figure 1G), the incidence of metastasis was similar between the KPC and KPC/TFF1KO mice (Figure 1H). In addition, the survival of these mice did not show any difference (Figure 1I). These results seem inconsistent with the traditional understanding of the nature of EMT; 
however, recent reports have suggested that EMT does not necessarily promote metastasis of the tumor in vivo ${ }^{5,6}$; thus, it appears that our findings are consistent with recent data on EMT.

TFF1 expression enhances the chemosensitivity of pancreatic cancer in humans and mice.

Given the above findings, we hypothesized that TFF1 might be associated with the chemosensitivity of pancreatic cancer. If TFF 1 inhibits EMT, the expression of TFF1 in tumor cells would increase the sensitivity of pancreatic cancer to chemotherapy. To investigate this hypothesis, we transfected the TFF1 overexpression vector into the pancreatic cancer cell line Panc1. Western blotting confirmed the expression of TFF1 induced by the vector, and EMT markers (Snail and Slug) were found to be suppressed in the TFF1-expressing Panc1 cells (Supplemental Figure 1A). The proliferative ability assessed by WST-1 assays did not show a difference between the control and TFF1-expressing cells. These cells were then treated with gemcitabine, and the cell number was substantially decreased in the TFF1-expressing cells (Supplemental Figure 1B). In addition, the rate of apoptotic cells was higher in the TFF1-expressing cells than the control cells (Supplemental Figure 1C and D), 
confirming that the cellular expression of TFF1 is associated with the chemosensitivity of pancreatic cancer cells.

To investigate the chemosensitivity of human pancreatic cancer, we assessed the expression of TFF1 in surgically resected human pancreatic cancer specimens. As we have reported previously, the positivity rate of TFF 1 in pancreatic cancer was almost $80 \%$, and positivity was not associated with the survival rate after curative surgical resection $^{15}$. This result was consistent with the findings of our mouse model showing similar survival of the KPC and KPC/TFF1KO groups. To further investigate the association of TFF1 expression and chemosensitivity, we analysed patients who suffered postoperative disease recurrence and underwent chemotherapy $(\mathrm{n}=46$; first-line chemotherapy included gemcitabine $(n=27)$, FOLFIRINOX $(n=3)$, gemcitabine/nabpaclitaxel $(\mathrm{n}=4)$, and $\mathrm{S} 1(\mathrm{n}=12))$ (Figure 2A). The expression of TFF1 was investigated in the resected specimen (Figure 2B), and the survival rate after the initiation of chemotherapy was analysed, revealing that the patients with positive expression of TFF1 showed a better survival rate than the patients with negative expression (Figure $2 \mathrm{C}, \mathrm{p}=0.012)$.

To further confirm the association of TFF1 expression and chemosensitivity, we treated the KPC and KPC/TFF1KO mice with gemcitabine. Pathologically, the 
tumors of the KPC mice were found to have higher necrotic areas than those of the KPC/TFF1KO mice (Figure 2D and E), and apoptotic cells evaluated by cleaved caspase 3 were found more frequently in the KPC mice than the KPC/TFF1KO (Figure 2F and $\mathrm{G})$. In addition, while the KPC mice showed improved survival when treated with gemcitabine, the KPC/TFF1KO mice did not (Figure 2H and I). These findings indicate that loss of TFF1 enhances the chemoresistance of pancreatic cancer in mice, which is completely consistent with human data.

Extracellular TFF1 enhances the chemosensitivity of pancreatic cancer cells in vitro

Although TFF1 expression in tumor cells is associated with chemosensitivity, TFF1 is a secreted protein, and it remains unclear whether TFF1 functions intracellularly or extracellularly. Although the precise mechanisms by which TFF1 functions have not yet been clarified, TFF1 might function in an autocrine or paracrine fashion. We thus hypothesized that TFF1 could display its effect even when administered extracellularly in the form of protein. To confirm this, we administered recombinant human TFF1 (rhTFF1) to Panc1 cells, and EMT status was investigated. As expected, the mRNA expression of the EMT markers SNAI1 and SNAI2 was significantly decreased by rhTFF1 treatment without the upregulation of 
TFF1transcription of the cells (Supplemental Figure 2), suggesting that TFF1 exerts its influence on cancer cells extracellularly.

These findings indicate that TFF1 administration might improve the chemosensitivity of pancreatic cancer and can be a therapeutic option when administered along with traditional chemotherapy; however, while the recombinant protein can be a therapeutic option for humans, the risk of biological contamination is not negligible. Thus, we chemically synthesized human TFF1 peptides (hereafter referred to as shTFF1) with conserved steric structures. Pancl cells were then treated with shTFF1 and gemcitabine to investigate their chemosensitivity. Similar to the results obtained with the TFF1-expressing vector, shTFF1 increased the rate of apoptotic cells induced by gemcitabine (Figure 3A, B). Cleaved caspase 3, an apoptotic marker, was also upregulated by combined treatment with gemcitabine and shTFF1 (Figure 3C). The relative cell number was decreased by gemcitabine, and shTFF1 enhanced this effect (Figure 3D). These results suggest that extracellularly administered shTFF1, as well as the cellular expression of TFF1, improves the chemosensitivity of pancreatic cancer cells. 
Clinically, repeated chemotherapy frequently results in tumor resistance, probably due to the induction of EMT in cancer cells. To investigate the association of gemcitabine-induced EMT and the role of shTFF1, we treated Panc1 cells with gemcitabine and/or shTFF1, and then, their molecular characteristics were analysed. Real-time PCR revealed that various EMT markers (SNAI1, SNAI2, VIM, CDH2, ACTA2, and ZEB1) were upregulated by gemcitabine, and this impact was counteracted by the addition of shTFF1 (Figure 4A). In contrast, epithelial markers (OCLN, TJP1, CLDN1, and CDH1) were downregulated by gemcitabine and reupregulated by the addition of shTFF1 (Figure 4B). In addition, Wnt target genes (EPHB3, TCF7, CTNNB1, ZCCHC12, and CCND1) showed a similar pattern as EMT markers (Figure 4C). Given that the Wnt pathway can induce EMT in cancer cells, these results indicate that shTFF1 functions to minimize the activation of the Wnt pathway induced by gemcitabine, thus suppressing EMT and eventually inducing chemosensitivity in pancreatic cancer.

To confirm these changes in protein expression, we performed western blotting using cell lysates treated as described above. The EMT markers (Snail, Slug, aSMA, and ZEB1) showed a similar pattern, in which upregulation by gemcitabine was cancelled by additional shTFF1 (Figure 5A). Conversely, epithelial markers (E- 
cadherin, Zo-1, claudin 1, and occludin) were downregulated by gemcitabine and reupregulated by addition of shTFF1, confirming the EMT characteristics of each group (Figure 5B). Notably, shTFF1 alone inhibited EMT, especially at the protein level, but not at the mRNA level. Considering that protein expression reflects long-term changes in mRNA levels, these results suggest that consecutive administration of shTFF1 can cause accumulative changes in cancer cells and results in stronger effects. For Wnt pathway activity, the phosphorylation status of $\beta$-catenin was investigated. Phosphorylation at Ser552 and Ser675, which are associated with the stabilization and accumulation of $\beta$-catenin in the nucleus, was upregulated by gemcitabine and downregulated by additional shTFF1 (Figure 5C). Phosphorylation at Ser33, Ser37, and Thr41, which indicates the destabilization of $\beta$-catenin, was increased by shTFF 1 treatment. In addition, phosphorylation of AKT, the downstream effector of Wnt signalling, was decreased in the shTFF1-treated group (Figure 5D). These results reconfirmed the function of shTFF 1 in inhibiting EMT and Wnt signalling induced by gemcitabine treatment.

TFF1 inhibits cancer stemness induced by gemcitabine in vitro 
We then hypothesized that the chemoresistance and EMT inhibited by TFF1 are closely associated with cancer stemness, and the stem cell markers of pancreatic cancer cell lines were investigated under gemcitabine and shTFF1 treatment. Panc1 cells were treated with gemcitabine and shTFF1, and then, the expression of CD133 was analysed by flow cytometry. As expected, while the proportion of CD133-positive cells was increased by gemcitabine treatment, it was decreased by the addition of shTFF1 (Figure 6A). This finding was consistent with the changes in EMT and Wnt signalling mentioned above. In terms of the cellular expression of TFF 1, Panc1 cells show relatively high expression of TFF1 among various human pancreatic cancer cell lines ${ }^{11}$. To explore the generality of the efficacy of shTFF1, we employed additional pancreatic cancer cell lines with relatively low TFF1 expression (PK9, KLM1, and KP4), and stem cell markers (CD133 and NANOG) were analysed by real-time PCR. Although the expression status and the reaction of these markers were different among cell lines, the stemness of the cells was generally enhanced by gemcitabine treatment and counteracted by the addition of shTFF1 (Figure 6B). These results confirmed that the availability of shTFF1 in pancreatic cancer treatment is not limited to those with high TFF1 expression, and a wide variety of pancreatic cancers would benefit from shTFF1 treatment. 
Combined treatment with gemcitabine and shTFF1 arrests tumor growth in vivo

To investigate the efficacy of shTFF1 in vivo and to explore the possible use of shTFF1 in pancreatic cancer treatment, we employed a xenograft mouse model. Panc1 cells were transplanted subcutaneously into nude mice, and the mice were treated with gemcitabine (intraperitoneally, twice per week, $40 \mathrm{mg} / \mathrm{kg}$ ) and/or shTFF1 (subcutaneously, twice per week, $1 \mu \mathrm{g} / \mathrm{mouse}$ ) (Figure 7A). Eventually, the tumors were harvested after 4 weeks of treatment (Figure 7B). Considering that TFF1 might function in a paracrine or autocrine fashion and that TFF 1 might be a physiologically active peptide, shTFF1 was administered subcutaneously according to the existing administration method of hormones such as insulin. While the volume of the tumor increased with time in the control mice, gemcitabine treatment inhibited this growth, and the additional shTFF1 significantly accelerated the efficacy of gemcitabine (Figure 7C). Of note, combined treatment with gemcitabine and shTFF1 almost arrested the growth of the tumor (Figure 7D), supporting the possibility of combined treatment with TFF1 and traditional chemotherapy.

TFF1 has an effect on spontaneously developed pancreatic cancer 
The efficacy of TFF1 was clearly demonstrated in a xenograft mouse model;

however, this model lacks the complicated microenvironment of pancreatic cancer, such as cancer-associated fibroblasts (CAFs), immune cells and microvessels. To further investigate the impact of gemcitabine/TFF1 treatment in vivo, we administered this treatment to a KPC mouse model of pancreatic cancer. In the absence of synthesized mouse TFF1, we employed recombinant mouse TFF1 (rmTFF1) instead. Gemcitabine and TFF1 were administered with the same protocol as in the xenograft model, starting at the age of 2 months old. As expected, gemcitabine alone improved the diseasespecific survival of the KPC mice, and rmTFF1 further improved this survival time, with median survival times of 3.4 months, 6.4 months, and 8.4 months (control, gemcitabine, and gemcitabine/TFF1 treatment, respectively) (Figure 8A). The mice treated with rmTFF1 did not show any signs of adverse events, and the body weight increased, similar to that in the gemcitabine alone group, suggesting that the additional TFF1 treatment was well tolerated (Figure 8B). The mice were harvested when they showed any sign of morbidity, and the tumors of these mice were analysed by immunohistochemistry, revealing that CD133 and ZEB1 expression was increased in the gemcitabine group but redownregulated in the gemcitabine/rmTFF1 group (Figure 8C-F). These results confirmed that the impact of TFF1 on EMT and stemness was 
reproducible in this mouse model. These results suggest the efficacy of gemcitabine/TFF1 treatment for pancreatic cancer, not only in the implantation model but also in the spontaneous cancer-developing model with the tumor microenvironment preserved. 


\section{Discussion}

In this study, we revealed that TFF1 acts as a tumor suppressor to inhibit EMT

and cancer stemness, thus enhancing the chemosensitivity of pancreatic cancer.

Importantly, extracellularly administered TFF1 exhibit a similar function as cellular expression of TFF1, indicating that TFF1 therapy might be a promising approach for pancreatic cancer treatment in the near future.

TFFs are secreted proteins predominantly expressed in the gastrointestinal mucosal epithelium; therefore, the investigation of TFFs has been limited in the area of gastrointestinal regeneration and carcinogenesis. We have investigated their functions in the pancreas and liver, revealing that TFFs are responsible for regeneration and carcinogenesis in these organs ${ }^{11,12,15-18}$. Interestingly, TFFs are expressed in the stem or progenitor cell niche and responsible for the regeneration of peripheral tissue $\mathrm{e}^{19,20}$, suggesting the association of TFFs and cellular stemness. The relationship between tissue stem cells and carcinogenesis has long been debated, and it seems that loss of control of proliferation and differentiation in the stem cell niche eventually causes the development of neoplastic diseases ${ }^{21,22}$. Considering this evidence, one can assume that the loss of TFFs in the stem cell niche results in the development of neoplastic lesions. Admittedly, the loss of TFF2 in the progenitor cell compartment of the pancreatic ductal 
epithelium resulted in the development of precancerous neoplasms ${ }^{18}$. The stemness of peripheral tissue cells and that of cancer cells does not necessarily match; however, it seems plausible that TFF1 is closely associated with the stemness of neoplastic cells, and it is consistent with the finding of this study that TFF1 inhibits stemness of pancreatic cancer.

TFFs are secreted proteins abundantly expressed in the gastrointestinal epithelium, and the expression of TFFs is associated with coordinated mucins ${ }^{23}$. It is easily comprehensible that TFFs are secreted into the gastrointestinal lumen with mucins to protect the mucosal epithelium; however, TFFs also exist in the bloodstream and urine ${ }^{24,25}$, suggesting that TFFs are secreted into the bloodstream as well as into the gastrointestinal lumen. If TFFs in the bloodstream harbor physiological activity and function in distant organs, TFFs might function not only in a paracrine or autocrine fashion but also in a hormonal fashion. In fact, subcutaneously administered TFF1 significantly suppressed tumor growth in vivo, indicating that TFF1 enters the bloodstream and then reaches tumor cells, where it functions as a tumor suppressor. Nevertheless, it remains to be clarified how secreted TFF1 functions in distant organs. One hypothesis is that TFF1 might act as a ligand to interact with a receptor of the cells. For instance, secreted frizzled-related proteins (SFRPs), which act as antagonists, bind 
to the Wnt receptor frizzled (FZD) and inhibit Wnt signalling ${ }^{26,27}$. TFF1 may function in a similar way to inhibit Wnt signalling and eventually suppress cancer stemness. Nevertheless, further investigations are needed to clarify the precise mechanisms of the tumor-suppressive role of TFF1.

The results of this study indicate that the tumor-suppressive role of TFF1 might be useful not only in cancer treatment but also in preventive therapy for malignant diseases. Although recent reports show the role of TFF1 as a tumor suppressor, this conclusion was primarily based on the finding that TFF1-deficient mice frequently develop malignant disease in the stomach, pancreas and liver. Deficiency of the TFF1 gene is congenital in these mice, and it is not clear whether TFF1 inhibits the initiation of neoplastic cells or tumor growth. This study clearly showed the efficacy of TFF1 in inhibiting the tumor growth of pancreatic cancer in a xenograft model; however, in the TFF1-treated KPC model, the injected TFF1 might have inhibited the development of neoplastic cells, presumably from the stem cell niche. If this is the case, TFF1 can be used for the prevention of disease development as well as cancer treatment. Some populations, such as those with chronic pancreatitis (for pancreatic cancer) and liver cirrhosis (for hepatocellular carcinoma), have a high risk of cancer development. To date, there is no effective treatment for these patients to prevent cancer development; 
thus, frequent imaging is the only option. Once preventive therapy by TFF1 is established, it might allow these patients to avoid future surgical treatment and chemotherapy.

In conclusion, we found that TFF 1 inhibits EMT and stemness in pancreatic cancer. The chemosensitivity of the tumor was increased by injected TFF1; thus, combined therapy with TFF1 and traditional chemotherapy could be useful in pancreatic cancer treatment. Further experiments are needed to reveal the precise mechanisms of TFF1 as a tumor suppressor in various organs. 
Author contributions: Conceptualization, Investigation, Methodology, Visualization, Writing, J.Y.; Resources, S.O.; Funding Acquisition, J.Y.; Data Curation, M.S., T.M., S.O., N.W. and A.O.; Supervision, Y.Y. and T.K.; and Project Administration, E.T.

Data availability: All data generated and analysed during the current study are included in this article and supplementary information files. The additional data that support the findings of this study are available from the corresponding author on reasonable request.

Grant Support: This work was supported by the Japan Society for the Promotion of Science (JSPS) and Grants-in-Aid for Scientific Research (KAKENHI) (grant numbers 17K10695 and 20H03751).

Disclosure: The authors have declared that no conflict of interest exists.

Declarations: All animal experiments were conducted in accordance with approval from the Institute for Laboratory Animal Research, Nagoya University Graduate School of Medicine (approved number; 20328), and with fundamental guidelines for proper conduct of animal experiment and related activities in academic research institutions 
(Ministry of Education, Culture, Sports, Science and Technology, Japan: Notice No. 71).

All methods are reported in accordance with ARRIVE guidelines for the reporting of animal experiments. 


\section{References}

1. Conroy, T. et al. FOLFIRINOX versus gemcitabine for metastatic pancreatic cancer. $N$ Engl J Med 364, 1817-1825 (2011).

2. Von Hoff, D.D. et al. Increased survival in pancreatic cancer with nab-paclitaxel plus gemcitabine. N Engl J Med 369, 1691-1703 (2013).

3. Marcucci, F., Stassi, G. \& De Maria, R. Epithelial-mesenchymal transition: a new target in anticancer drug discovery. Nat Rev Drug Discov 15, 311-325 (2016).

4. Iwatsuki, M. et al. Epithelial-mesenchymal transition in cancer development and its clinical significance. Cancer Sci 101, 293-299 (2010).

5. Zheng, X. et al. Epithelial-to-mesenchymal transition is dispensable for metastasis but induces chemoresistance in pancreatic cancer. Nature 527, 525-530 (2015).

6. Fischer, K.R. et al. Epithelial-to-mesenchymal transition is not required for lung metastasis but contributes to chemoresistance. Nature 527, 472-476 (2015).

7. Kalluri, R. \& Weinberg, R.A. The basics of epithelial-mesenchymal transition. JClin Invest 119, 1420-1428 (2009).

8. Wang, X. et al. Identification of a novel function of TWIST, a bHLH protein, in the development of acquired taxol resistance in human cancer cells. Oncogene 23, 474-482 (2004).

9. Thiery, J.P., Acloque, H., Huang, R.Y. \& Nieto, M.A. Epithelial-mesenchymal transitions in development and disease. Cell 139, 871-890 (2009).

10. Polyak, K. \& Weinberg, R.A. Transitions between epithelial and mesenchymal states: acquisition of malignant and stem cell traits. Nat Rev Cancer 9, 265-273 (2009).

11. Yamaguchi, J. et al. Trefoil factor 1 inhibits epithelial-mesenchymal transition of pancreatic intraepithelial neoplasm. J Clin Invest 128, 3619-3629 (2018).

12. Yamaguchi, J. et al. Pancreatic duct glands (PDGs) are a progenitor compartment responsible for pancreatic ductal epithelial repair. Stem Cell Research 15, 190-202 (2015).

13. Mejlvang, J. et al. Direct repression of cyclin D1 by SIP1 attenuates cell cycle progression in cells undergoing an epithelial mesenchymal transition. Mol Biol Cell 18, 4615-4624 (2007).

14. Lovisa, S. et al. Epithelial-to-mesenchymal transition induces cell cycle arrest and parenchymal damage in renal fibrosis. Nat Med 21, 998-1009 (2015).

15. Sunagawa, M. et al. Trefoil factor family 1 expression in the invasion front is a poor prognostic factor associated with lymph node metastasis in pancreatic cancer. Pancreatology 17, 782-787 (2017).

16. Hayashi, Y. et al. Loss of trefoil factor 1 inhibits biliary regeneration but accelerates the 
hepatic differentiation of progenitor cells in mice. Biochemical and biophysical research communications 506, 12-19 (2018).

17. Ochiai, Y. et al. Trefoil Factor Family 1 Inhibits the Development of Hepatocellular Carcinoma by Regulating beta-Catenin Activation. Hepatology 72, 503-517 (2020).

18. Yamaguchi, J. et al. Loss of Trefoil Factor 2 From Pancreatic Duct Glands Promotes Formation of Intraductal Papillary Mucinous Neoplasms in Mice. Gastroenterology 151, 1232-1244 e1210 (2016).

19. Thiem, S. et al. Inducible gene modification in the gastric epithelium of Tff1-CreERT2, Tff2-rtTA, Tff3-luc mice. Genesis 54, 626-635 (2016).

20. Quante, M., Marrache, F., Goldenring, J.R. \& Wang, T.C. TFF2 mRNA transcript expression marks a gland progenitor cell of the gastric oxyntic mucosa. Gastroenterology 139, 2018-2027 e2012 (2010).

21. Blanpain, C. Tracing the cellular origin of cancer. Nat Cell Biol 15, 126-134 (2013).

22. White, A.C. \& Lowry, W.E. Refining the role for adult stem cells as cancer cells of origin. Trends Cell Biol 25, 11-20 (2015).

23. Longman, R.J. et al. Coordinated localisation of mucins and trefoil peptides in the ulcer associated cell lineage and the gastrointestinal mucosa. Gut 47, 792-800 (2000).

24. Radon, T.P. et al. Identification of a Three-Biomarker Panel in Urine for Early Detection of Pancreatic Adenocarcinoma. Clin Cancer Res 21, 3512-3521 (2015).

25. Aikou, S. et al. Tests for serum levels of trefoil factor family proteins can improve gastric cancer screening. Gastroenterology 141, 837-845 e831-837 (2011).

26. Suzuki, H. et al. Epigenetic inactivation of SFRP genes allows constitutive WNT signaling in colorectal cancer. Nat Genet 36, 417-422 (2004).

27. White, B.D., Chien, A.J. \& Dawson, D.W. Dysregulation of Wnt/beta-catenin signaling in gastrointestinal cancers. Gastroenterology 142, 219-232 (2012). 
Figure 1: TFF1 inhibits EMT in pancreatic cancer in vivo. (A, C, E) Representative IHC images of Ki67, Snail and ZEB1 in PDAC developed in the KPC and KPC/TFF1KO mice. Scale bars; 100 mm. (B, D, F) Quantification of the positive cells shown as the labeling index. ${ }^{* *} \mathrm{p}<0.01(\mathrm{G})$ Representative microscopic images of distant metastasis found in the liver and lung. Scale bars; 200 mm. (H) The incidence of distant metastasis in each mouse model. (I) Overall survival of each mouse model.

Figure 2: TFF1 expression enhances the chemosensitivity of pancreatic cancer in humans and mice. (A) Schema of patient selection. (B) Representative IHC images of TFF1-positive and TFF1-negative PDAC. Scale bars; $100 \mu \mathrm{m}$. (C) Overall survival of the patients after chemotherapy. (D) Representative microscopic image of PDAC found in the KPC and KPC/TFF1KO mice treated with gemcitabine. Scale bars; $200 \mu \mathrm{m}$. (E) Quantification of the necrotic area shown as a percentage. ${ }^{*} \mathrm{p}<0.05$ (F) Representative IHC image of cleaved caspase 3 in each mouse model. Scale bars; $100 \mu \mathrm{m}$. (G) Quantification of cleaved caspase 3-positive cells shown as the labeling index. ${ }^{*} \mathrm{p}<0.05$ (H) Overall survival of the gemcitabine-treated KPC mice. (I) Overall survival of the gemcitabine-treated KPC/TFF1KO mice. 
Figure 3: shTFF1 enhances the chemosensitivity of pancreatic cancer cells in vitro.

(A, B) The cells were treated with gemcitabine $(1 \mu \mathrm{M})$ and/or shTFF1 $(100 \mathrm{ng} / \mathrm{ml})$, and then, the rate of apoptotic cells was assessed by a Muse ${ }^{\mathrm{TM}}$ Annexin V \& Dead Cell Kit.

(C) The expression of caspase 3 and cleaved caspase 3 in each group assessed by western blotting. (D) Relative cell number was evaluated by WST-1 assays. $* * p<0.01$

Figure 4: shTFF1 inhibits gemcitabine-induced EMT and the Wnt pathway in vitro. The cells were treated with gemcitabine $(1 \mu \mathrm{M})$ and/or shTFF1 $(100 \mathrm{ng} / \mathrm{ml})$, and then, the relative gene expression of EMT markers (A), epithelial markers (B), and Wnt target genes (C) was assessed by RT-PCR. ${ }^{*} \mathrm{p}<0.05,{ }^{*} \mathrm{p}<0.01$.

Figure 5: shTFF1 inhibits gemcitabine-induced EMT and the Wnt pathway in protein level in vitro. Protein expression levels of EMT markers (A), epithelial markers (B), $\beta$-catenin phosphorylation (C), and AKT phosphorylation (D) were assessed by western blotting.

Figure 6: shTFF1 inhibits cancer stemness induced by gemcitabine in vitro. (A) Flow cytometric image of Panc1 cells treated with gemcitabine $(1 \mu \mathrm{M})$ and/or shTFF1 (100 
$\mathrm{ng} / \mathrm{ml}$ ). (B) The expression of cancer stem cells (CD133 and NANOG) in pancreatic cancer cells (Panc1, PK9, KLM1, and KP4) treated with gemcitabine and/or shTFF1 assessed by RT-PCR. ${ }^{*} \mathrm{p}<0.05,{ }^{* *} \mathrm{p}<0.01$

Figure 7: Combined treatment with gemcitabine and shTFF1 arrests tumor growth in vivo. (A) Schema of the schedule of mouse treatment. (B) Gross appearance of implanted tumors harvested after 4 weeks of treatment. (C) Quantification of the tumor volume in the mice treated with gemcitabine and shTFF1. ${ }^{*} p<0.05, * * p<0.01$. (D) Time course of tumor volume in each group of mice.

Figure 8: Recombinant TFF1 enhances the chemosensitivity of pancreatic cancer in mice. (A) Disease-specific survival of the KPC mice treated with gemcitabine and rmTFF1. (B) Average body weight of each group of mice treated with gemcitabine and/or rmTFF1. (C) Representative IHC image of CD133 in mouse tumors. Scale bars; $100 \mu \mathrm{m}$. (D) Representative IHC image of ZEB1 in mouse tumors. Scale bars; $100 \mu \mathrm{m}$. (E) Quantification of CD133 positivity shown as a percentage. (F) Quantification of ZEB1positive cells shown as a labelling index. ${ }^{*} \mathrm{p}<0.05,{ }^{*} \mathrm{p}<0.01$. 
Supplemental Figure 1: Overexpression of TFF1 suppresses EMT and increases chemosensitivity of pancreatic cancer cells in vitro. (A) Protein expression of TFF1, Snail, and Slug in the cells transfected with the control and TFF1-overexpressing plasmids assessed by western blotting. (B) Relative cell number of each transfected cell line treated with gemcitabine $(1 \mu \mathrm{M})$ for 72 hours. ${ }^{* *} \mathrm{p}<0.01(\mathrm{C}, \mathrm{D})$ The rate of apoptosis of each group was assessed by the Muse ${ }^{\mathrm{TM}}$ Annexin V \& Dead Cell Kit.

Supplemental Figure 2: rhTFF1 suppresses EMT in pancreatic cancer cells in vitro. The relative expression of TFF1, SNAI1, and SNAI2 in the cells treated with rhTFF1 assessed by RT-PCR. * ${ }^{*}<0.01$ 
A

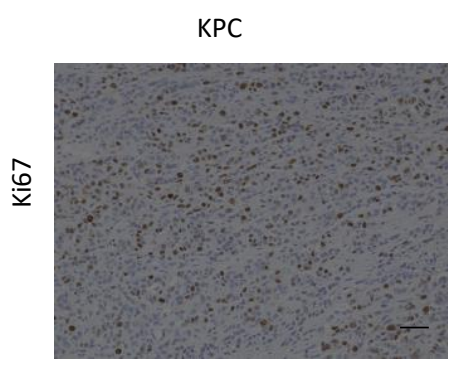

C

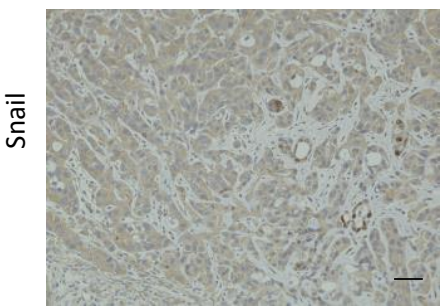

E

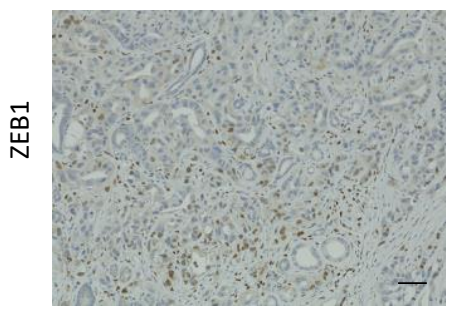

G

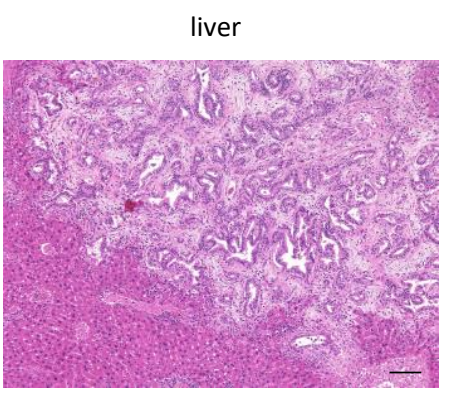

KPC/TFF1KO
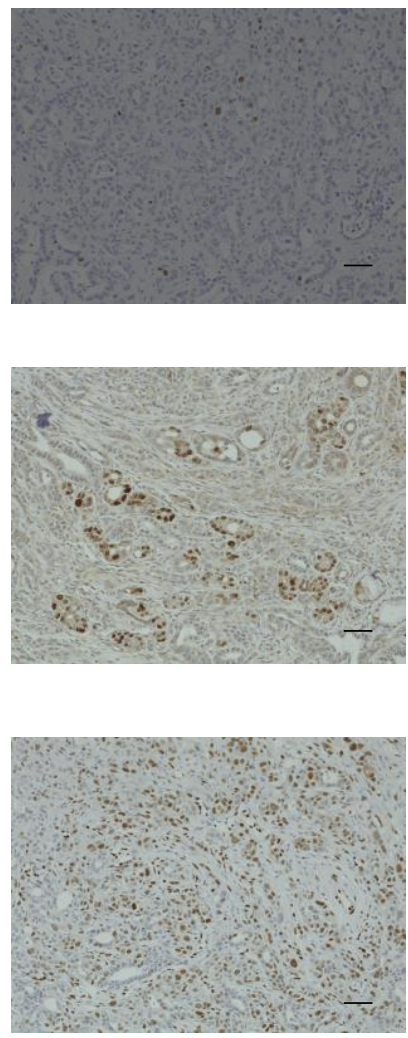

lung
B

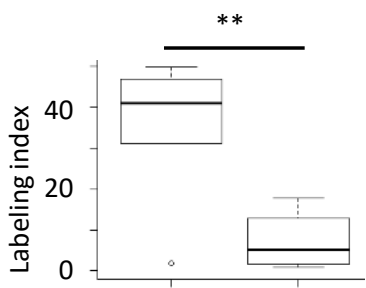

Figure 1
D

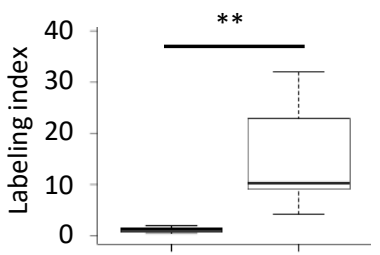

F

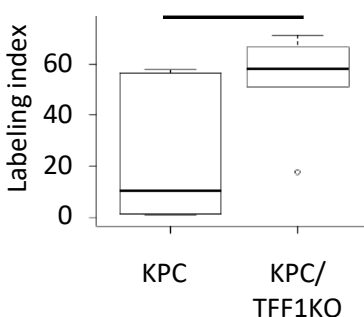

$\mathrm{H}$

Incidents of distant metastasis

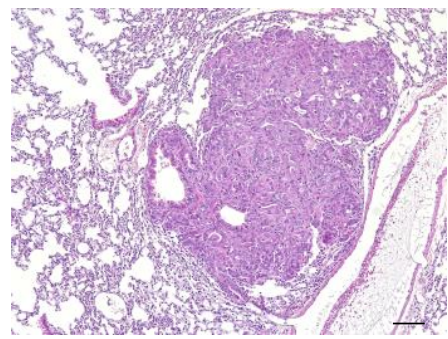

KPC $22.2 \%(n=4 / 18)$ KPC/TFF1 $\% \quad 28.6 \%(n=4 / 14)$

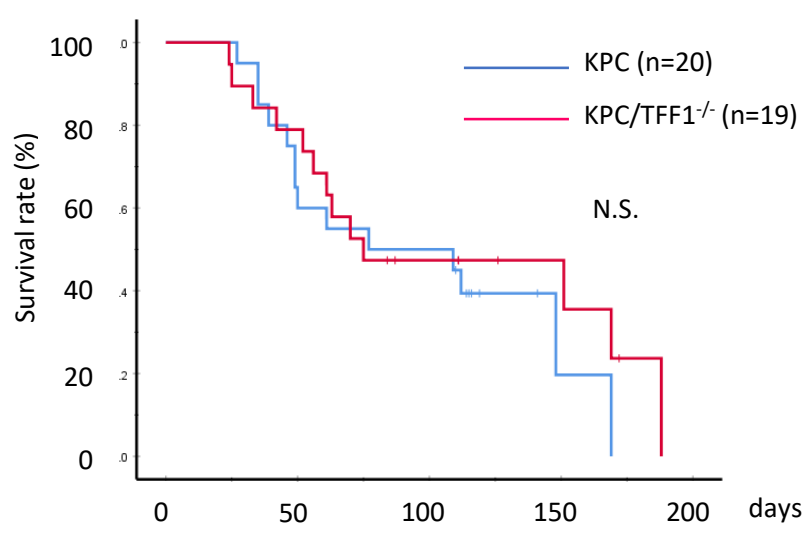


A

TFF1-positive $(n=38)$

B

TFF1-positive

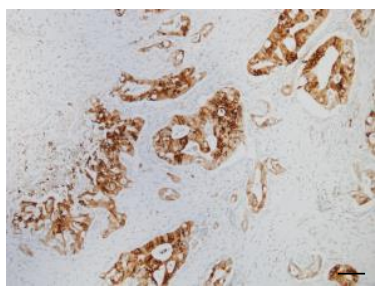

KPC

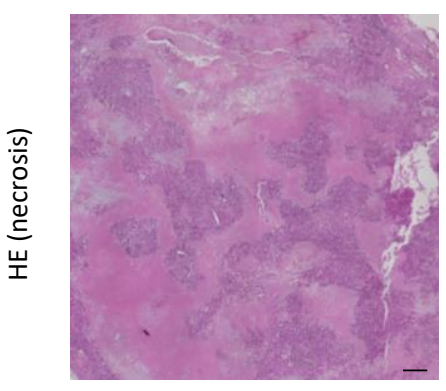

F

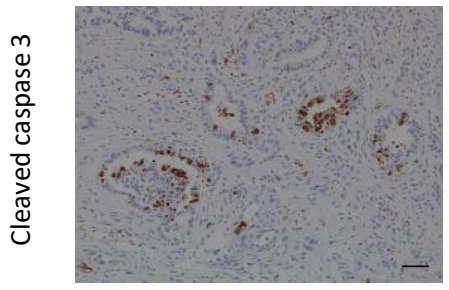

TFF1-negative

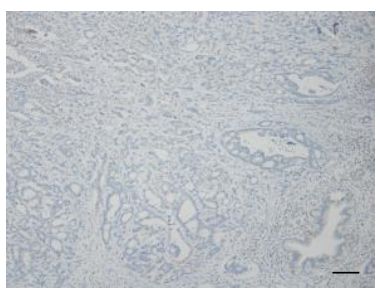

KPC/TFF1KO
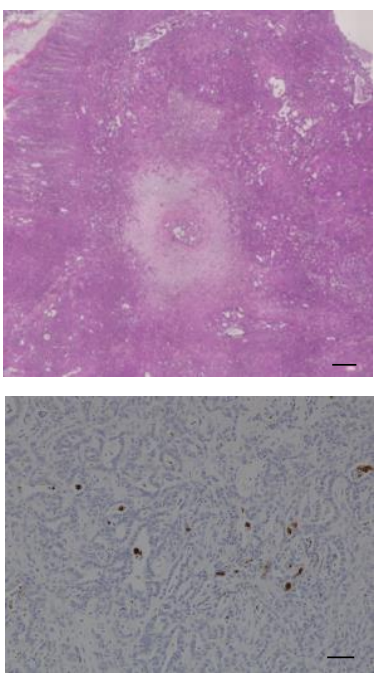

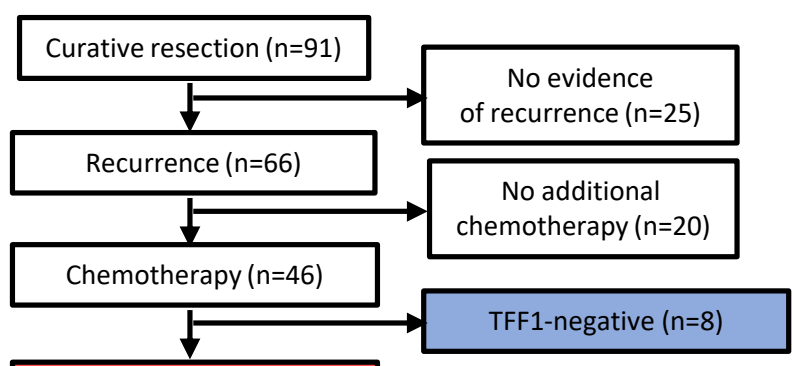

C

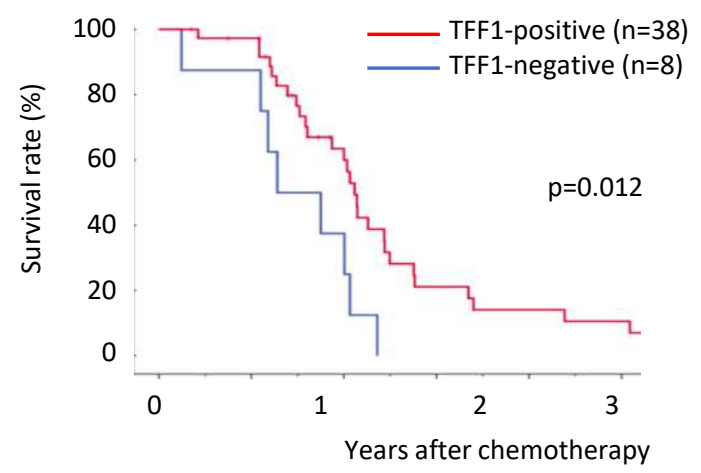

Number at risk

\begin{tabular}{lcccc}
\hline Positive & 38 & 20 & 4 & 2 \\
Negative & 8 & 3 & 0 & 0
\end{tabular}

E

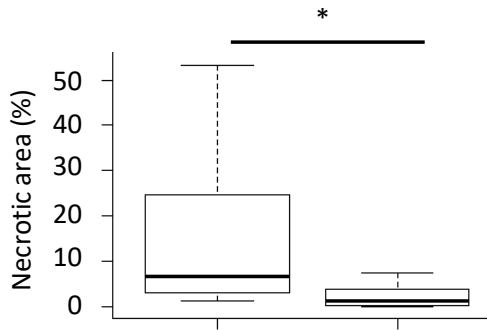

G

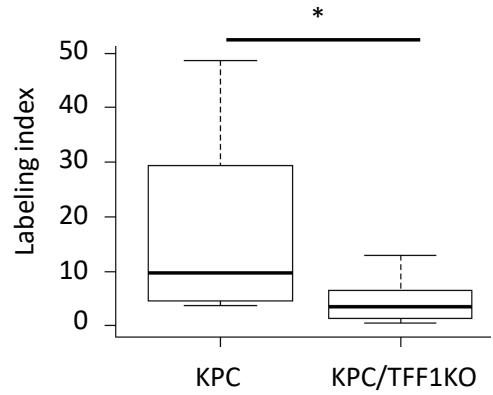

H 部

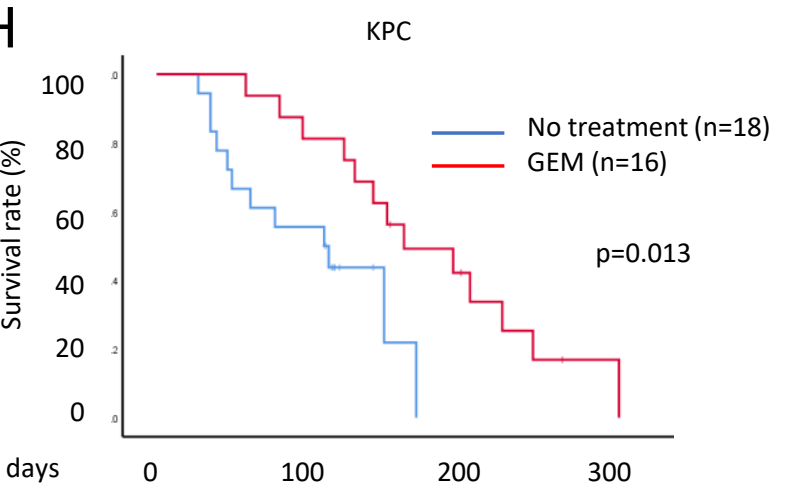

I

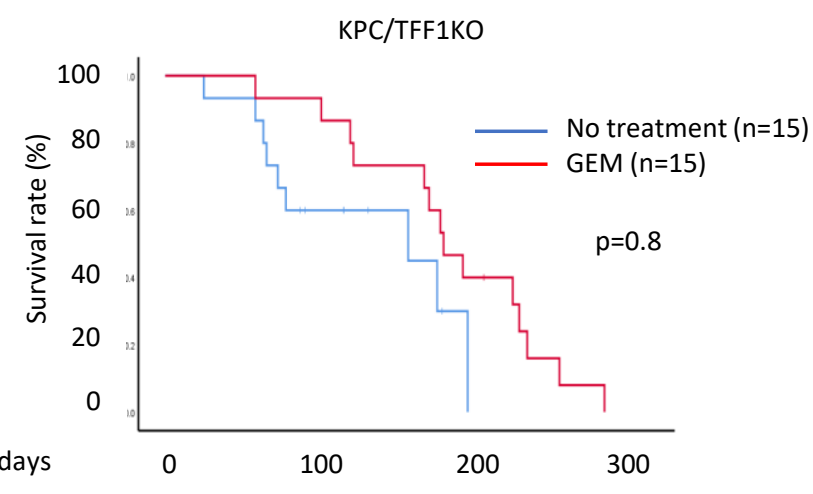


A
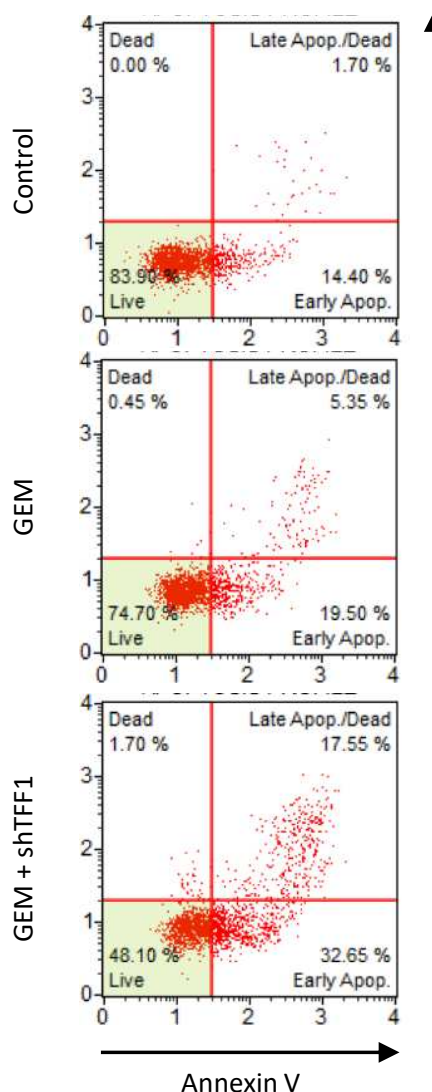

题

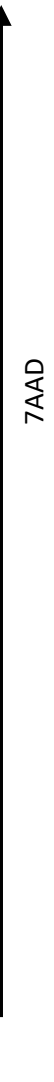

B

C

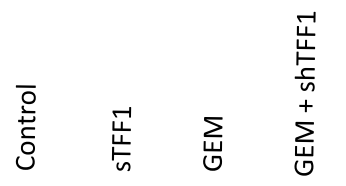

Caspase 3

Cleaved caspase 3

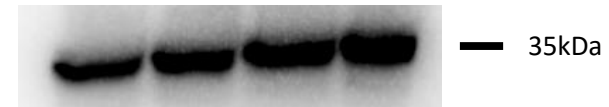

$\beta$-actin

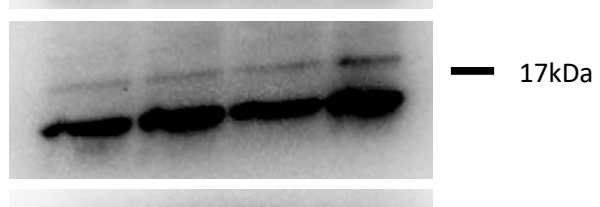

$48 \mathrm{kDa}$
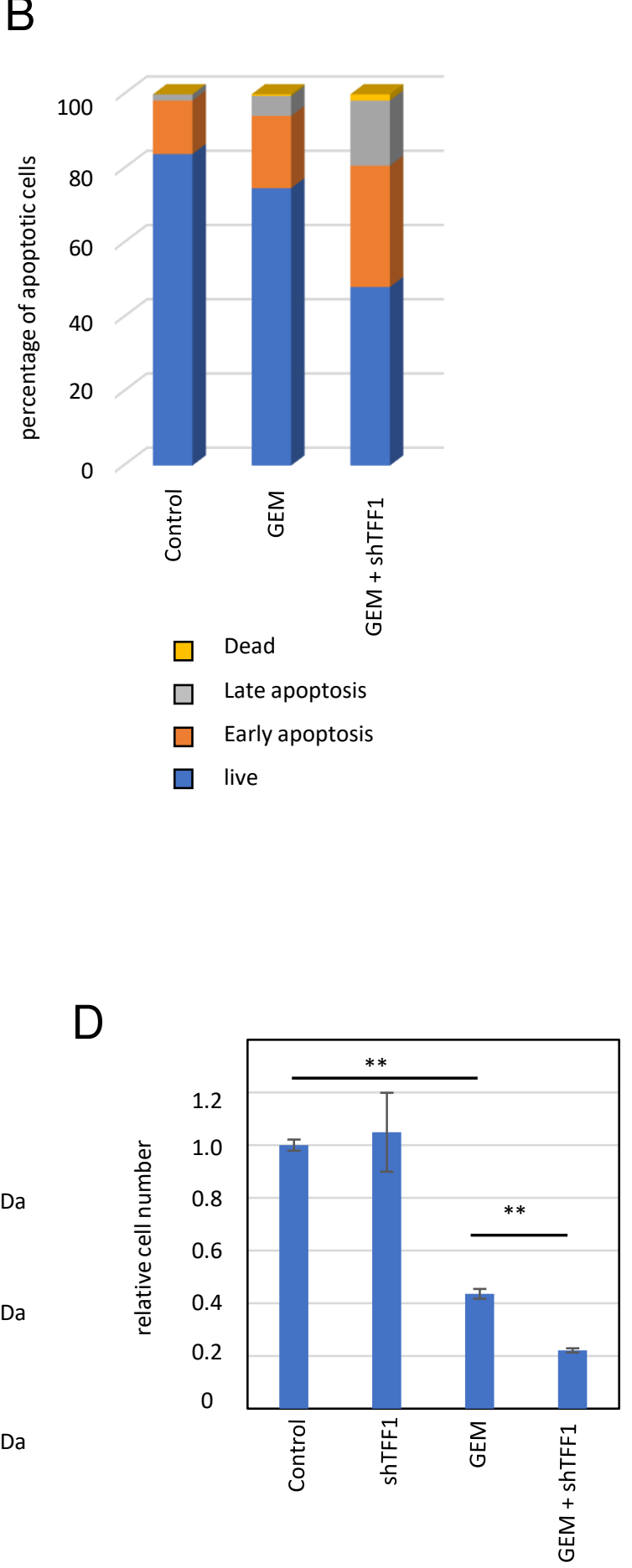
A
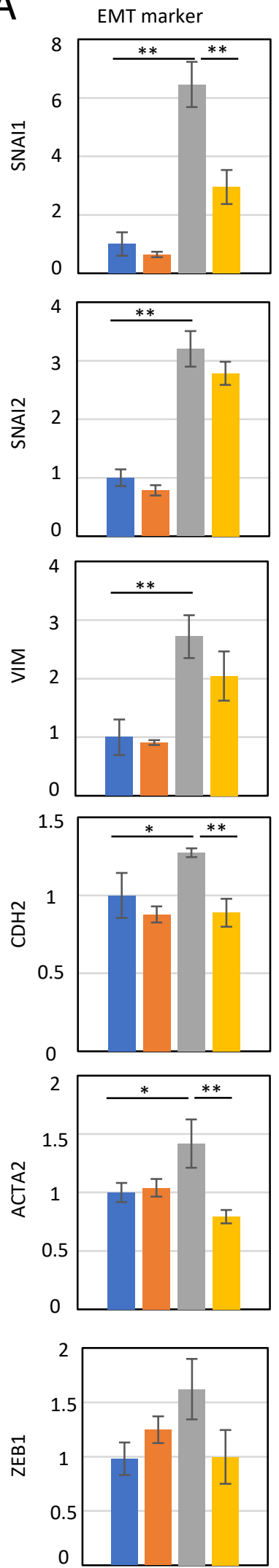
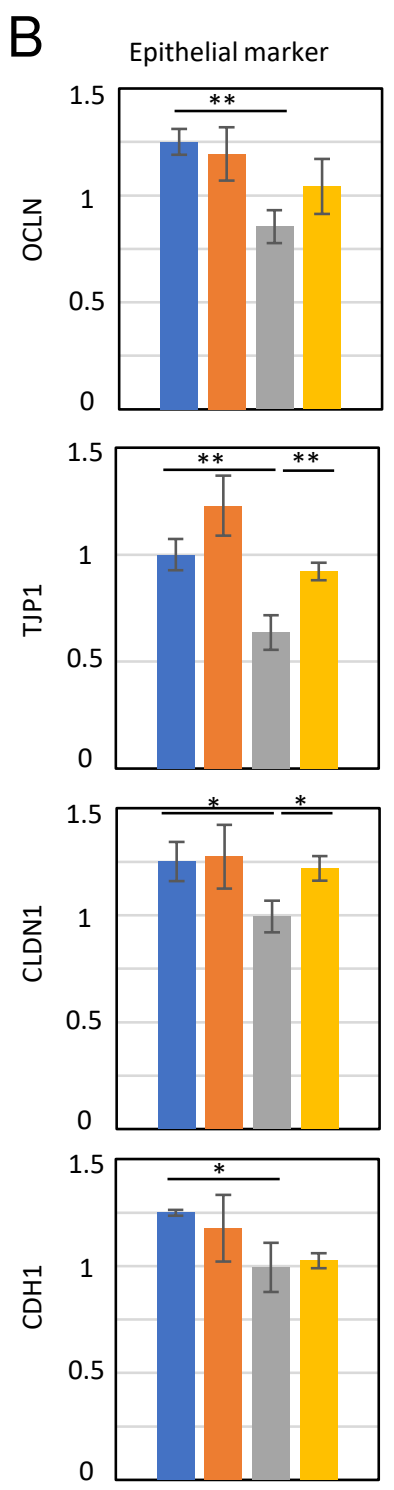

$\square$ Control

$\square \quad$ shTFF1

$\square$ Gemcitabine

$\square$ Gem+shTFF1
C
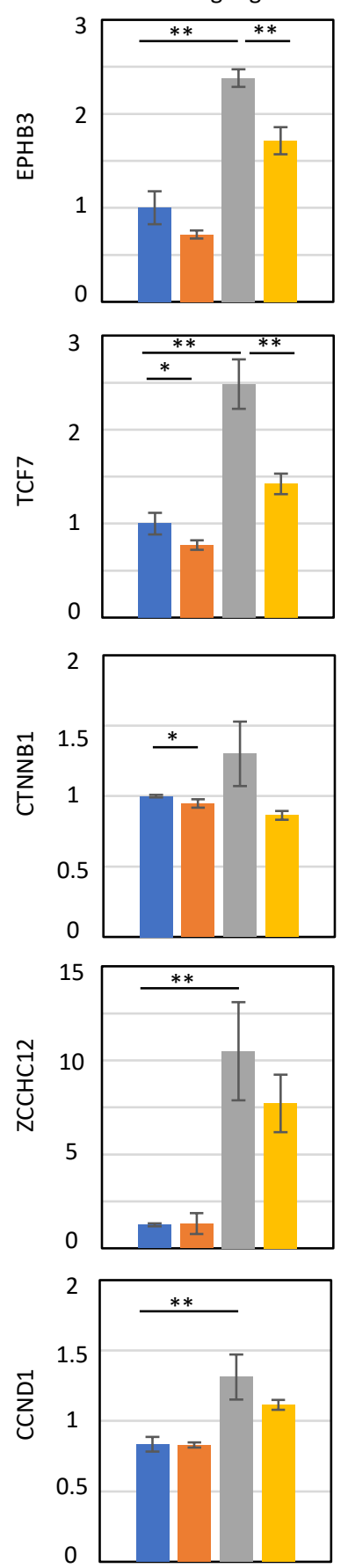
A

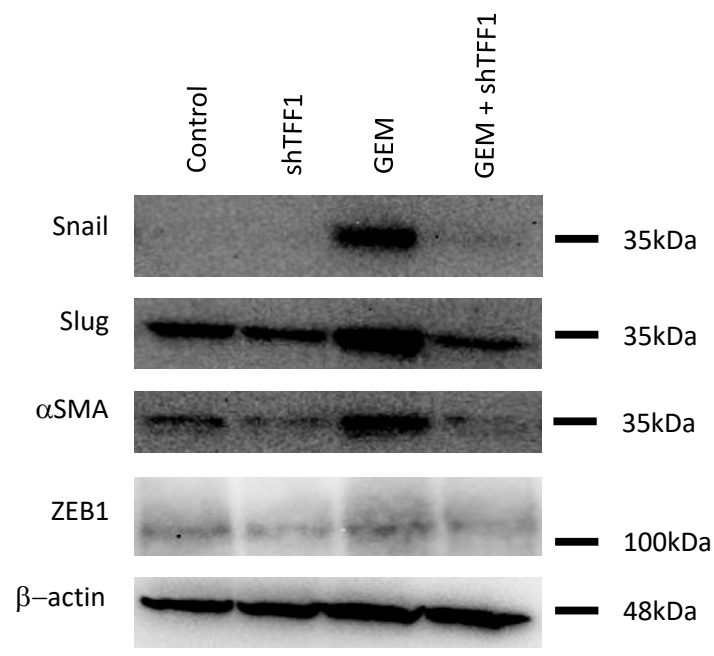

C

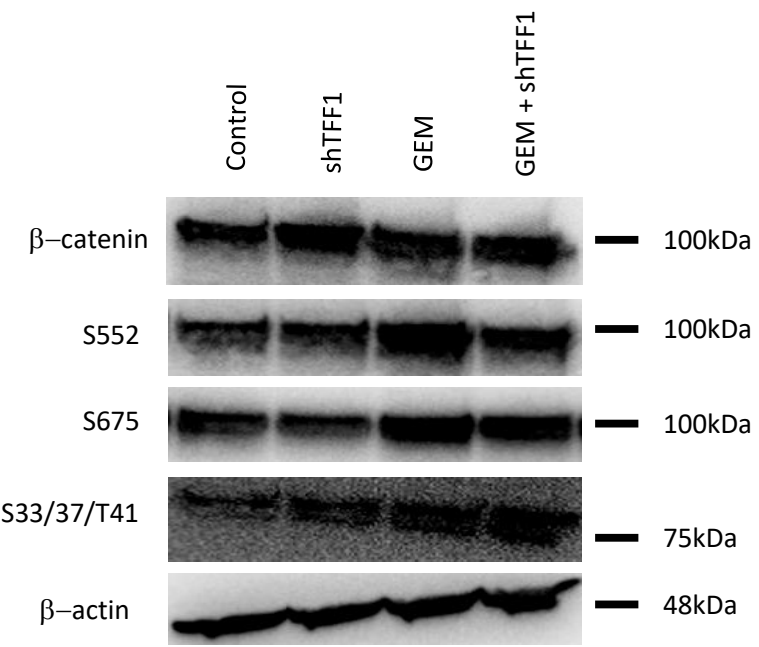

B

Figure 5

D

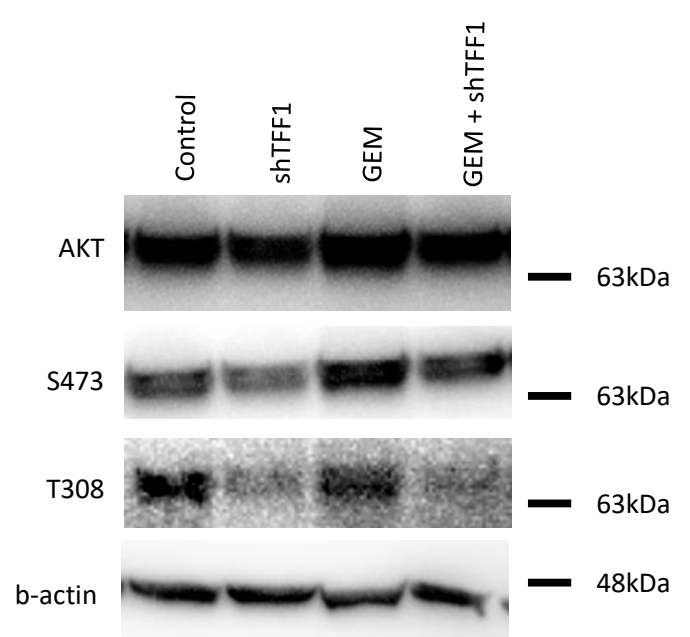


A

control

ShTFF1

gemcitabine

Gem + shTFF1

Figure 6
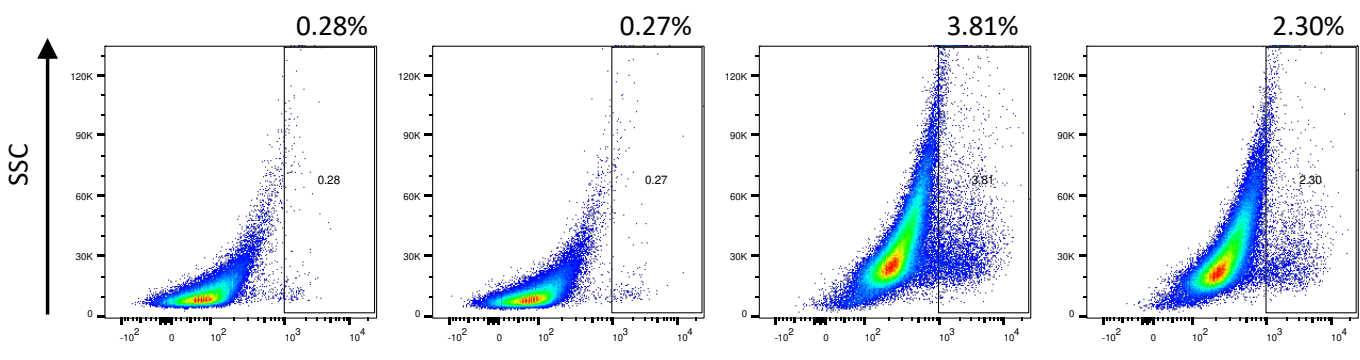

APC (CD133)

B
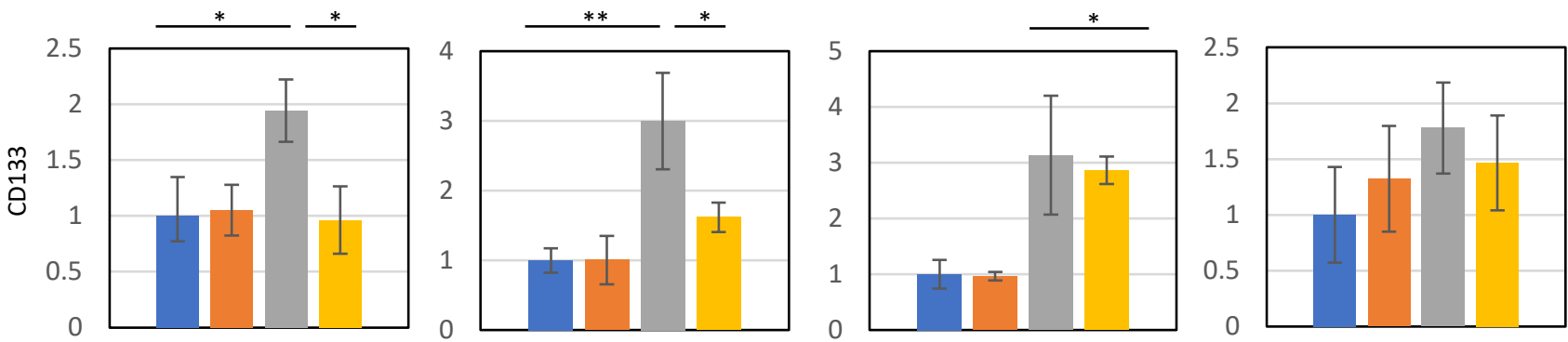

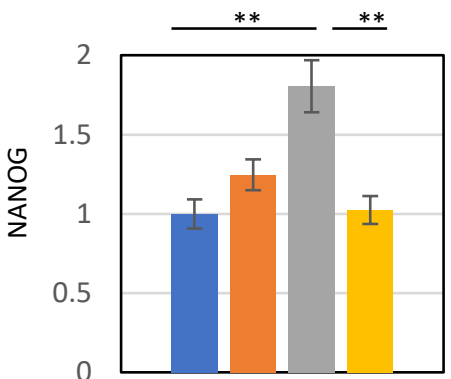

panc1

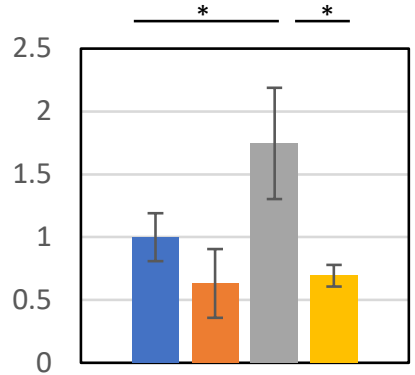

PK9

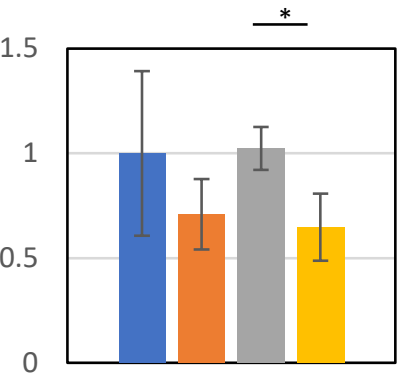

KLM1

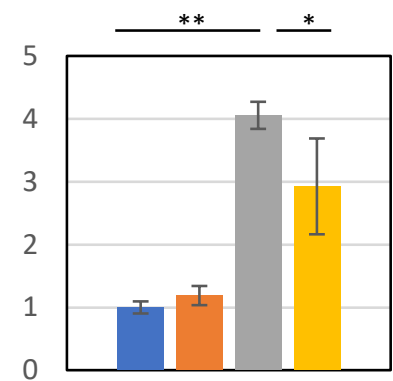

KP4
Control
口 STFF1
ㅁ Gemcitabine
ㅁ Gem+sTFF1 


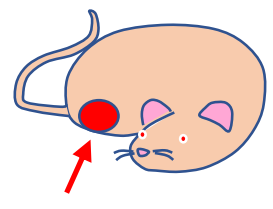

Nude mouse

GEM 40mg/kg (i.p., twice/week)

sTFF1 $1 \mu \mathrm{g} /$ body (s.c., twice/week)

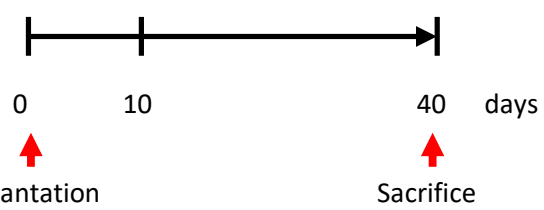

B

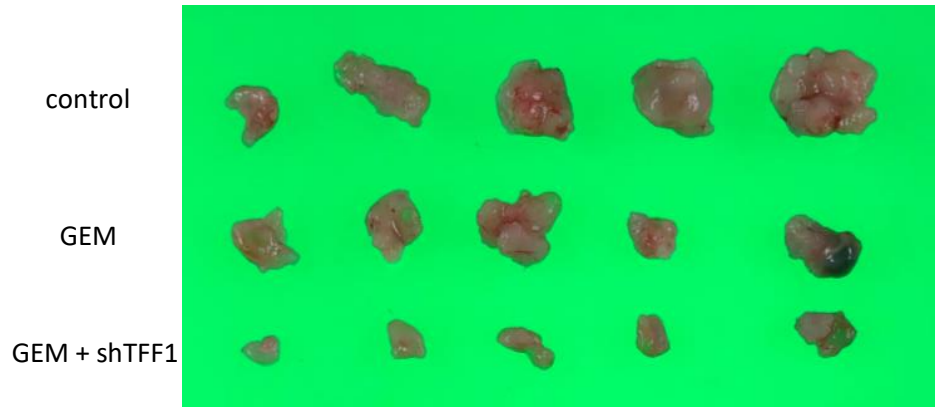

D
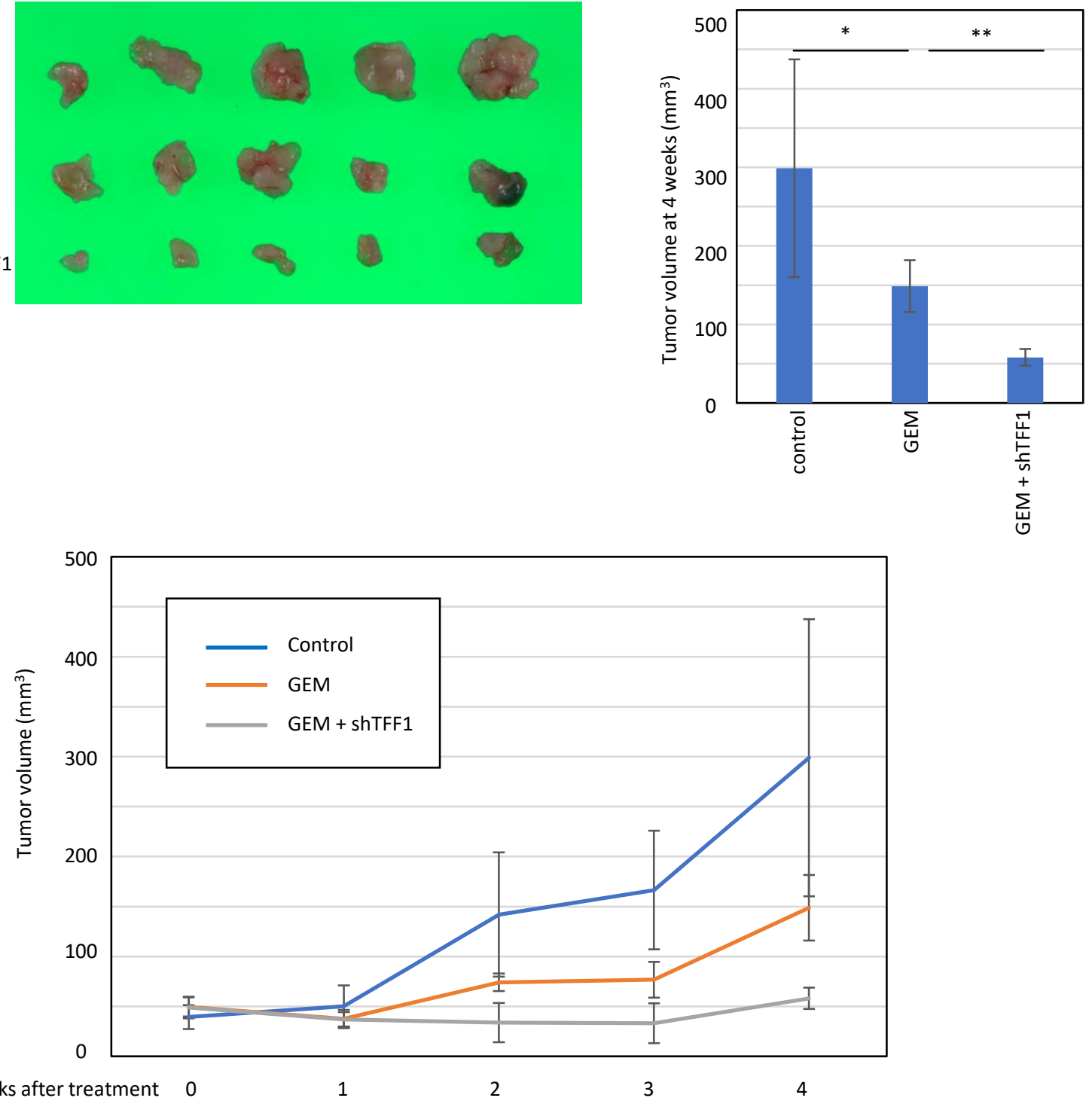

Weeks after treatment implantation

C 
A

B

Figure 8
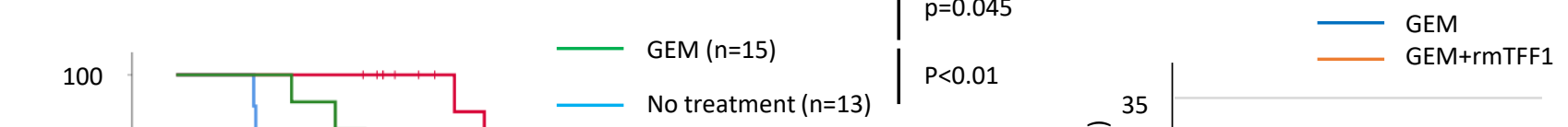

C

No treatment

GEM

GEM+rmTFF1
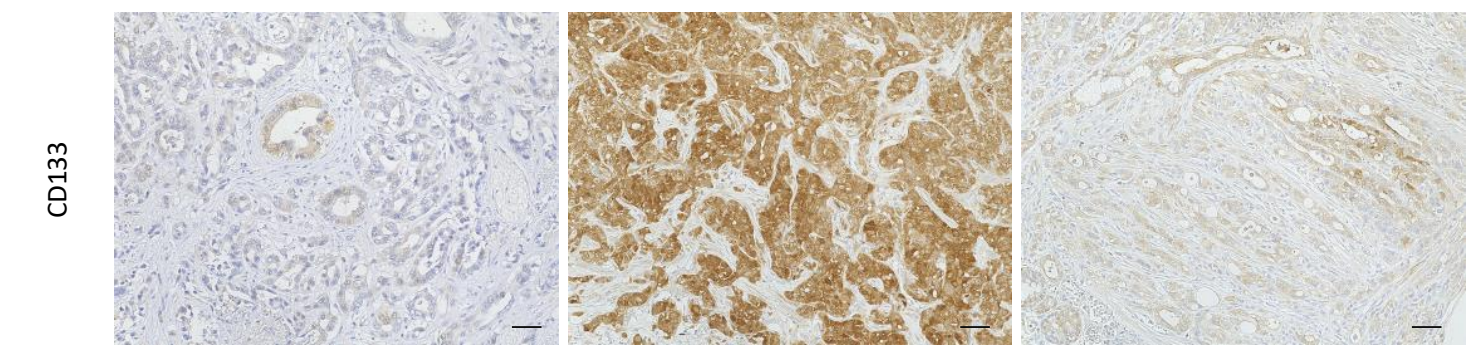

D
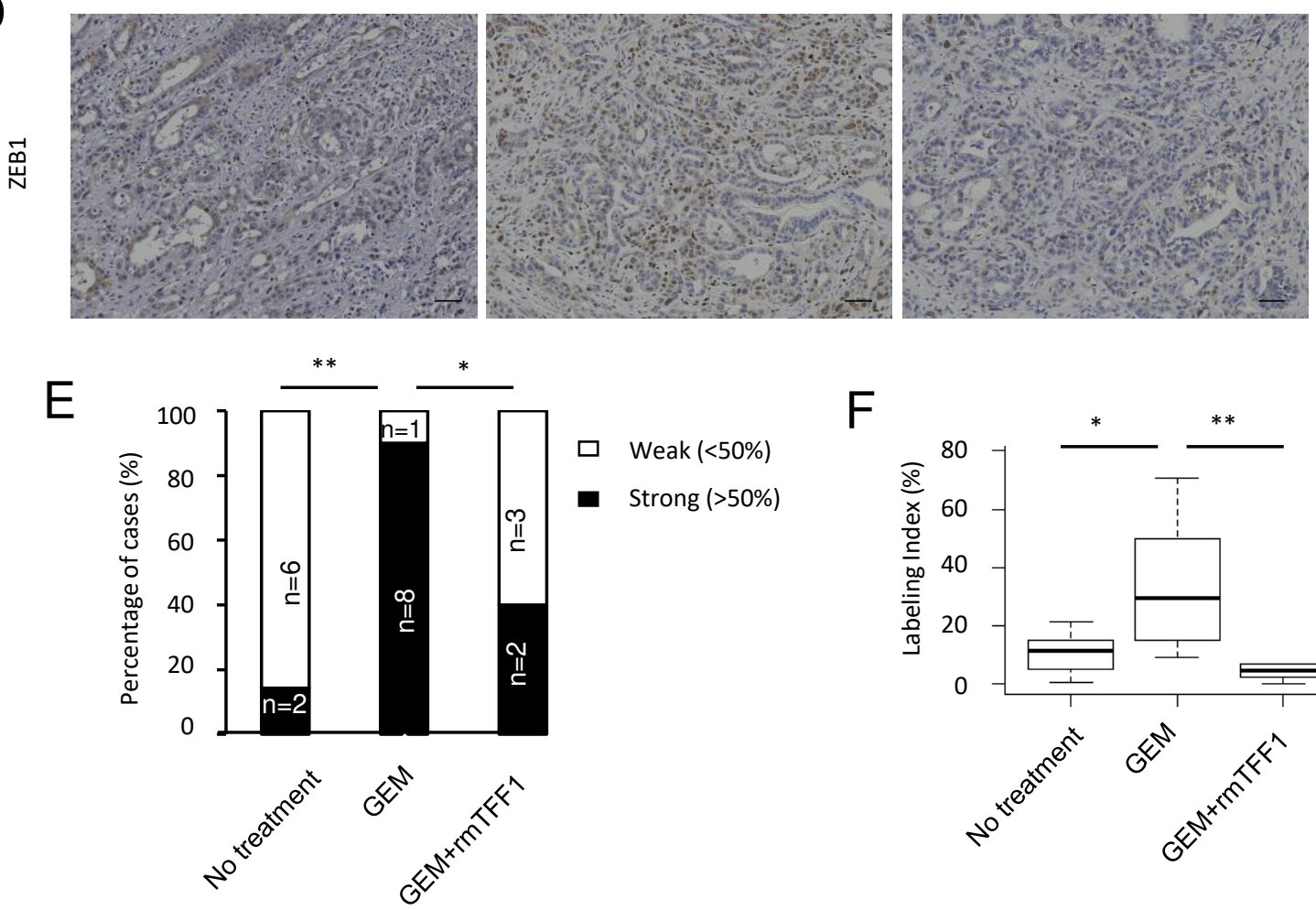

F

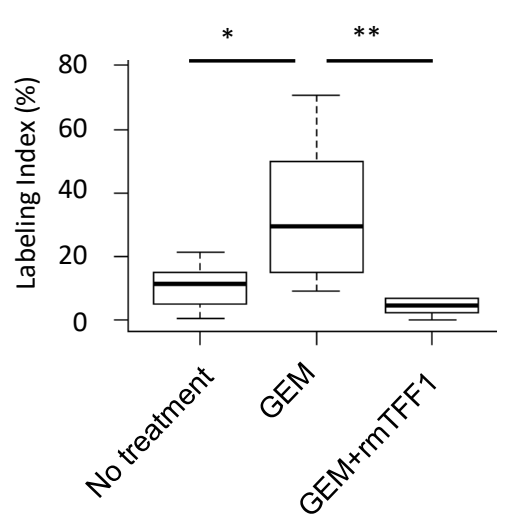




\section{Supplementary Files}

This is a list of supplementary files associated with this preprint. Click to download.

- Spplementalfiles.pdf 